\title{
Integrated Analysis of the IncRNA/circRNA-miRNA- mRNA Expression Profiles Reveals Novel Insights Into Potential Mechanisms in Response to Root- knot Nematodes in Peanut
}

\section{Ping Xu}

Linyi University

Hui Li

Linyi University

Xiaohua Wang ( $\square$ wangxiaohua19880721@126.com)

Linyi University

\section{Ge Zhao}

Linyi University

Xiaofei Lu

Linyi University

\section{Shengjie Dai}

Linyi University

\section{Xiaoyu Cui}

Linyi University

\section{Mei Yuan}

Ministry of Agriculture, Shandong Peanut Research Institute

Zhengning Liu

Linyi University

\section{Research Article}

Keywords: Peanut, Root-knot nematode, Molecular mechanism, Competing endogenous RNA, Regulatory network

Posted Date: November 8th, 2021

DOl: https://doi.org/10.21203/rs.3.rs-968829/v1

License: (9) This work is licensed under a Creative Commons Attribution 4.0 International License. Read Full License 
Version of Record: A version of this preprint was published at BMC Genomics on March 28th, 2022. See the published version at https://doi.org/10.1186/s12864-022-08470-3. 


\section{Abstract}

\section{Background}

The high content of oil and protein makes peanut the main oil and edible crop in the world. Root-knot nematode forms root-knot by infecting peanut roots, which lead to poor development of peanut roots and seriously restricts the yield of peanut in the world. With the release of peanut genome, a large number of genetic loci controlling peanut root-knot nematode have been detected, but the molecular mechanism of root-knot nematode is still unclear.

\section{Results}

The whole transcriptome RNA-seq was used to reveal the divergent response to root-knot nematode stress in peanut roots. A total of 430 mRNAs, 111 miRNAs, 4453 IncRNAs and 123 circRNAs were identified differential expression between infected and no-infected peanut, respectively. To understand the potential mechanisms in response to root-knot nematodes in peanut roots, the expression profiles of IncRNA/circRNA-miRNA-mRNA network were constructed. A total of 10 IncRNAs, 4 circRNAs, 5 miRNAs and 13 mRNAs can regularly the expression of mRNA during root-knot nematodes stress by forming competing endogenous RNA and participate in oxidation-reduction process and other various biological metabolism pathways in peanut. The results gained will reveal the role of ceRNAs of peanut in response to root-knot nematodes.

\section{Conclusion}

The GO classification and KEGG pathway enrichment analysis of core regulatory networks revealing the ceRNAs participate in oxidation-reduction, peroxidase activity, lignin synthesis in xylem and flavonoid synthesis process. Overall, those results could gain the knowledge of the role of no-coding RNAs in response to root-knot nematodes.

\section{Background}

Root-knot nematode (Meloidogyne incognita) is one of the most harmful plant parasitic nematodes in the world [1]. For a long time, the control methods of root-knot nematode mainly depended on chemical insecticides, crop rotation and host plant resistance [2]. Among them, the use of chemical insecticides (such as dibromochloropropane) plays an important role in the prevention and treatment of root-knot nematode disease, but it also causes serious environmental pollution [3]. In addition, due to the spacious feeding range of nematodes in the plant kingdom, the effect of crop rotation on nematode control is not ideal [4]. Therefore, it is of profound significance to improve the resistance of crops to root-knot nematode disease.

Peanut is an important oil crop, and the rooting nematode disease is one of the most destructive diseases in peanut production areas worldwide [5]. At present, the cultivation and planting of disease-resistant 
peanut varieties has become one of the most effective ways to control root-knot nematode disease. Since the resistance of peanut cultivars to root-knot nematode is weak at present, and no effective peanut cultivars against root-knot nematode have been found [3]. The regulation mechanism of peanut against root-knot nematode is complex, and many genetic loci were detected in previous studies [6]. However, what the role of RNAs played in peanut root-knot nematode traits was still unclear.

RNA is formed by condensation of ribonucleotides through phosphoric acid two ester bonds. According to the structure and function, the RNA can be divided into messenger RNA (mRNA) and non-coding RNA (ncRNA) [7]. Based on molecular weight, non-coding RNAs are mainly divided into long non-coding RNAs (IncRNA), circular RNAs (circRNAs) and small RNAs (miRNA) [8-9]. MiRNAs bind to mRNA through complete or incomplete base complementation, and regulate the expression of target genes by inhibiting translation or directly leading to mRNA degradation [10]. However, the competing endogenous RNA including IncRNA and circRNA which contain the miRNA binding region could relieve the inhibition of miRNA on target genes (mRNA) [11]. The application of next-generation sequencing technology greatly facilitated the discovery of IncRNAs, circRNA and miRNA in plants [7, 9]. Based on the regulation of genes from the post transcriptional level, the ncRNA played a key role in plants in response to biotic and abiotic stresses such as diseases and pests, temperature and drought [12]. The miR472-RDR6 could silence the pathway modulated PAMP through posttranscriptional control of the disease resistance in Arabidopsis [13]. The over-expression of miR160a and miR398b enhanced resistance to Magnaporthe oryzae resistance in Oryza sativa [14]. The expression of mi172a was inhibited upon Aspergillus flavus infection in Arachis hypogaea [15]. A total of 347 cirRNAs controlling different flowering time were detected in peanut [16]. There were 1583 heat stress-specific circRNAs were identified in Arabidopsis [17]. There were 481 and 545 IncRNA showed differential expressions under dark treatments and different blue light treatments in Arabidopsis, respectively [18].In Chinese cabbage, the number of differentially expressed heat-responsive IncRNAs was 1229 [19]. A total of 40 differently expressed IncRNAs involved in nitrogen acquisition in maize root were identified [20]. The competing endogenous (ce) RNA hypothesis has gained the potential regulatory mechanism of in plant stress resistance. There were miRNA-regulated network involving 8834 mRNAs, 117 IncRNAs and 78 miRNAs were reconstructed in maize [21]. The potential ceRNAs network which contains 33 miRNA and 186 IncRNAs was constructed in chilling resistance of tomato [9]. A ceRNA including three IncRNAs (IncR9A, IncR117 and IncR616) and miR398 is reported to improve cold resistance of winter wheat [22]. The ceRNAs regulatory network consisted of one miRNA (osamiR156a_L + 1), two mRNA and 13 IncRNAs were observed enrich the potential regulatory mechanism of glyphosate-tolerant [23].

In the present study, cDNA and RNA libraries from the root-knot caused by nematodes (treatment) and the same area parts of root tissues (control) in peanut (Huayu 22) were constructed. A total of 430 mRNAs, 111 miRNAs, 4453 IncRNAS and 123 cirRNAs showed differential expression between infected and uninfected by root-knot nematode of peanut tissues. The ceRNAs network based on the interaction between mRNAs, IncRNAs, cirRNAs and miRNAs was constructed. That network could increase the knowledge of mechanisms in response to root-knot nematodes, improving edible oil and improving edible plant tissues for human and livestock. 


\section{Materials And Methods}

\section{Plant materials and phenotype evaluation}

The seed of the peanut Huayu22 (It was approved by Shandong crop variety Approval Committee of China in March 2003. https://baike.so.com/doc/5049390-5276442.html) was grown in the planting pot in the greenhouse in Linyi, China $\left(117.24^{\circ} \mathrm{E}, 34.22^{\circ} \mathrm{N}\right)$ from May of 2019 to May of 2020. The soil was derived from the experimental area where the root-knot nematode was seriously bursts at Qingdao, China $\left(120.41^{\circ} \mathrm{E}, 36.39^{\circ} \mathrm{N}\right)$. The root- knot nematode was growth and reproduction in living tomato plants. Rootknot nematodes were propagated by planting tomato ([9]). The infected tomato roots were sterilized and the eggs were collected by wet sieving. After mixed the root-knot nematodes eggs and sandy soil, the peanut seed was planted. Trial management followed standard breeding pot protocols. Peanuts grow under $16 / 8$ light photoperiod at $25^{\circ} \mathrm{C}$. After germination 21 days (five-leaf stage seeding), the peanut samples with root-knot nematodes were collected and counted. Three independent biological replicates samples which pooled from different plants were carried in this study. The sample which collected for miRNAome and transcriptome analysis was kept at $-80^{\circ} \mathrm{C}$ freezer, and the total RNA was isolated using the Trizol method [30-31]. Statistical analyses were performed with the SPSS software package (SPSS, statistics), and the one-way analysis of variance (ANOVA) was used to determine statistical difference [31].

\section{The whole transcriptome sequencing and data analysis}

Total RNA-seq offers the most comprehensive whole transcriptome analysis. After ligating RNA 3' and 5' adapter, the RNA libraries (miRNA and ribosome free strand-specific RNA sequencing library) were constructed through TruSeq RNA Sample Prep Kits (Illumina, San Diego, USA). Prepared libraries were sequence on the Illumina Hiseq 2500 platform (LC Science, Lianchuan Biotechnology Co., Ltd, Hangzhou, China). According to the Illumina protocol, the $2 \times 150$ bp paired-end reads and the $1 \times 50$ bp single-end reads were generated for mRNAs, IncRNAs, cirRNAs and miRNAs $([9,15])$.

The high quality reads with $<5 \%$ missing nucleotides, the length of sequence $>18 \mathrm{nt}$ and no continuous dimer nucleotides were selected $([9,32]$. The clean RNA-seq reads were mapped to the peanut (Arachis hypogaea L.) genome (http://peanutgr.fafu.edu.cn/index.php) using software of TopHat version 2.1.1. After removing the tRNA, scRNA, snoRNA and rRNA in GenBank non-coding RNA database, the clean reads were assembled and merged to final transcriptome using software of Cufflinks version 2.2.1 and Cuffmerge version 2.1.1. The software of minreap (http://sourceforge. net/projects/mireap) were carried in predicting the hairpin structures of miRNA precursors [9].

\section{Differently expression mRNA, IncRNAs, cirRNAs and miRNA identification}

After final transcriotome was produced, the differentially expressed mRNA between root-knot nematodes infected of peanut root and no-infected same tissues were identified based on the rigorous criteria of $\mathrm{P}<$ 0.01, FDR $<0.01$ and $\mid \log _{2}($ FPKM_Treat/ FPKM_CK) $\mid>2$ [31]. The differentially expressed IncRNA were 
identified with criteria: longer than $200 \mathrm{bp}$, coding potential calculator $<0$ and coding non coding index $<$ $0, P<0.01, Q<0.01$ and $\mid \log _{2}$ (FPKM_Treat/ FPKM_CK) $\mid>2$. The differentially expressed cirRNAs were identified based on the criteria of $\mathrm{P}<0.01$ and $\mid \log _{2}$ (SRPBM_Treat/ SRPBM_CK)|>2 [9]. The criteria of differential expressed miRNAs were identified with length from 18 to 25 nucleotides, $\mathrm{P}<0.01$ and $\| \log _{2}$ (RPM_Treat/ RPM_CK)|>2 [15].

\section{Target prediction and CE network construction}

The target of ncRNAs was determined through co-expression, genetic co-location and free energy forming secondary structures between IncRNAs, cirRNAs, miRNA and mRNA. After sequence blasting and free energy calculating, the IncRNA and cirRNA were identified as a candidate target ncRNAs of mRNA ([9]). The potential association between miRNAs and mRNAs was determined through miRTarBase (http://mirtarbase.mbc.nctu.edu.tw/), TargetScan (http://www.Targetscan.org/) and RNA22 (https://cm.Jefferson.edu/rna22/) [33]. Based on the above relationship, the ceRNA network was constructed and visualized by software of Cytoscape V 3.6.1.

\section{GO and KEGG enrichment analysis}

To analyze the biological functions of mRNAs and IncRNAs, GO classification and KEGG pathway enrichment analysis were carried by the topGO R packages and KOBAS software, respectively. With $p$ value $<0.01, \mathrm{GO}$ annotations will be divided into three categories (cellular component, molecular function and biological process).Moreover, biological signal pathways of mRNAs and IncRNAs could be revealed through the KEGG pathway database with threshold of false discovery rate $(F D R)<0.05$ [33].

\section{Results}

\section{Different peanut root morphology types caused by root-knot nematodes}

Compared with no-infected peanut, the Huayu22 infected by root-knot nematode showed significant at root node number (21.33 $\pm 4.16 v s 0, p=0.00089)$ (Fig.1, Fig.2a, Table 1). At the same time, the root-knot nematodes could cause short primary root length $(25.06 \pm 1.79$ vs 28.26 $\pm 1.38, p=0.0013)$ (Fig.2c, Table 1), decreased total root surface area $(644.94 \pm 21.53$ vs $478.36 \pm 42.96, p=0.0039)$ (Fig.2d, Table 1), narrower root angle (79.26 $\pm 7.47 v s 106.58 \pm 8.94, p=0.015)$ (Fig.2e, Table 1), less lateral root density $(0.48 \pm 0.026$ vs $0.69 \pm 0.021, p=0.00041$ ) (Fig.2e, Table 1) but more lateral root number $(52.33 \pm 5.13 v s 41 \pm 3, p=0.029)$ in infected peanut (Fig.1, Fig.2b, Table 1).

\section{The differential expression mRNA in response to peanut root-knot nematode}

After investigated the whole genome gene expression profile of peanut in response to root-knot nematodes, a total of 430 differential expression mRNAs (427 up-regulated and 3 down-regulated) were identified in response to peanut root-knot nematode (Fig. S2a, Table S1.). The length of differential expressed mRNA was range from 200bp to 12283 bp (with an average of 1672 bp) (Fig. 3c, Table S1). 
Differentially expressed genes were distributed on 20 chromosomes. The number of differential expressed mRNAs (with an average of 21.5) was ranging from 11 on A02 chromosome to 35 on A01 and A03 chromosomes. The number of differential expressed genes was close in sub-A genome (213) and sub-B genome (217) (Fig S1). The functional annotation of differential expressed genes (329 out of 430) showed related to stress adaptation, such as heat shock cognate protein, cytochrome P450, pathogenesis-related protein, peroxidase and WRKY and MYB transcription factor (Table S1). The top GO annotation of differential expressed mRNAs was including defense response, oxidation-reduction process, signal transduction, response to wounding, nucleus, protein binding et al (Table S1).

\section{The differential expression miRNAs and their target genes}

To reveal the role of miRNA in response to root-knot nematodes, the expression profiles of miRNAs and target genes were identified. The length of differential expressed miRNA was ranged from 18 to 25 (with

an average of 21.75) nucleotide (Fig.3, Table S2). A total of 80 miRNAs showed differential expressed between infected and no-infected by root-knot nematode. In addition, 36 out of 80 showed up-regulated in response to root-knot nematodes (Fig.S2, Table S2).

A total of 1771 mRNAs were the target genes of those 80 miRNAs. There were 111 out of 1771 target genes showed differential expressed in response to root-knot nematodes (Table S3). The 111 target genes annotation were disease resistance-like protein DSC1, peroxidase and WRKY transcription factor et al. According to the GO and KEGG analysis, target genes were involved in a series of adversity response processes, such as, plant-pathogen interaction, MAPK signaling pathway-plant and starch and sucrose metabolism (Table S3). The mRNAs-miRNAs regulatory networks contained miRNA (gma-MIR482cp5_2ss12GA19CT) and mRNA (CTM7LX, JF37M9, NEIN3W, X5NWFC and Z9NEHU); miRNA (PC-3p30685_85) and mRNA (AJ79N4, NK4UTA, R13KY7 and XA3BQV); miRNA (PC-3p-14080_193) and mRNA (42IH8X, DQ3LYR and S1GD6Q) were constructed (Fig.4a, Fig.S3).

\section{CircRNAs acts as the sponge of miRNAs in response to peanut root-knot nematode}

A total of 123 differential expressed circRNAs (with 60 up-regulated and 63 down-regulated) were detected in response to peanut root-knot nematodes (Fig.S2c, Fig.S4). From 1 on A03 chromosome to 15 on A09 chromosome (with an average of 6.15), the number of circRNAs is unevenly distributed on each chromosome (Fig.S4). A total of 6 (with 2 up-regulated and 4 down-regulated) out of 123 differential expressed circRNAs were predicted bind to 7 miRNAs (with 2 up-regulated and 5 down-regulated). The GO and KEGG analysis showed that the differential expressed circRNAs involving in defense response, response to oxidative stress, response to temperature stimulus et al (Table S4). The circRNAs-miRNAs regulatory networks were constructed by circRNAs (circRNA113 and circRNA442) and miRNA (gmamiR10420_L+1R-1); circRNAs (circRNA226) and miRNA (PC-3p-14080_193); circRNAs (circRNA320) and miRNA (gma-MIR482c-p5_2ss12GA19CT); circRNAs (circRNA43) and miRNA (ptc-miR393a-3p) (Fig.4b, Fig.S3).

The role of IncRNAs played in regulatory ceRNA under root-knot nematode stress 
A total of 4453 significantly differentially expressed IncRNAs were identified by false discovery rate (FDR) $<0.05$, among which 2909 differentially expressed IncRNAs were up-regulated, and 1544 differentially expressed IncRNAs were down-regulated (Fig.S2d, Table S5). The length of differentially expressed IncRNAs were ranged from 200 to 552581 (most of IncRNAs were 200-300 nucleotide) nucleotide (Fig 3d). There were 13 differential expressed IncRNAs (6 up-regulated and 7 down-regulated) bind to 6 miRNAs (with 3 up-regulated and 3 down-regulated). The IncRNAs-miRNAs regulator interactions were constructed by IncRNAs (MSTRG.12823, MSTRG. 17002, MSTRG.33245 and MSTRG.42738) and miRNA (PC-3p-14080_193 and PC-3p-30685_85); IncRNAs (MSTRG.2115, MSTRG.30601, MSTRG.30599 and MSTRG.31962) and miRNA (gma-MIR482c-p5_2ss12GA19CT); IncRNAs (MSTRG.3150 and MSTRG.37521) and miRNA (mtr-miR319a-3p_R+1) (Fig.4d, Fig S3).

\section{The regulatory ceRNA network of IncRNA/circRNA-miRNA-mRNA in response to root-knot nematode stress}

The whole genome of differential expressed IncRNA, circRNA, mRNA and miRNA were detected (Fig.3, Fig.S2, Table S1-S7). The key IncRNA/circRNA-miRNA-mRNA competing endogenous RNA tetraploid subnetwork associated with root-knot nematode stress response was reconstruction (Fig.4, Fig. S3, Table S3, Table S6, Table S7). The first sub-network contained 6 IncRNAs (MSTRG.41742, MSTRG.43398, MSTRG.47397, MSTRG.55943, MSTRG.56279 and MSTRG.58883), 2 circRNAs (circRNA113 and circRNA442), 3 miRNAs (gma-miR10420_L+1R-1, vvi-MIR3630-p5_2ss19AG20CT_1 and PC-5p-7845_316) and 4 mRNAs (LMT1MP, J7B49U, 322B5E and K6N5RU). A total of two IncRNAs, one circRNA and two mRNA showed up-regulated in response to peanut root-knot nematode. Meanwhile, the number of IncRNAs, circRNAs, miRNAs and mRNAs showed down-regulated under root-knot nematode stress were four, one three and two, respectively. The second sub-network contains four IncRNAs (all down-regulated), one circRNA (down-regulated), one miRNA (up-regulated) and five mRNAs (three up-regulated and two donw-regulated). The final sub-network was constructed based on one down-regulated circRNA, one upregulated miRNA and four mRNAs (two up-regulated and two down-regulated) (Fig.5, Fig.S3). Based on the $\mathrm{GO}$ and KEGG analysis, the RNAs in ceRNA network was involved in peroxidase activity, lignin biosynthetic process and oxidation-reduction process (Fig.6, Fig.S5).

\section{Discussion}

Root-knot nematodes are major agricultural damaging pest worldwide [1]. After infected by root-knot nematodes, a series of physiological and biochemical were changed such as caused root tissue necrosis, affected peanut root activity, weakened peanut photosynthetic intensity and respiratory intensity [24]. However, what the role played in peanut root morphology were rarely studies. In present studies, the peanut which planted in root-knot nematodes environment were short primary root length, small total root surface area, narrow root angle, less lateral root density but more lateral root number than that in no rootknot nematodes soil (Fig. 1, Fig. 2). After infected with root-knot nematodes, the peanut root formed the root-knot and not extend. The roots are the most important organs for absorbing nutrients and water. The root-knot prevents peanut from absorbing nutrients. In order to absorb more nutrients, peanut roots 
developed more lateral roots after being infected by root-knot nematode [25]. At the same time, the mobility of root-knot nematodes is weak. Therefore, whether the root-knot nematode is evenly distributed in the soil is crucial for peanut disease. In order to quickly incubate root-knot nematodes, we bred nematodes by tomato infection. After that, the nematode was extracted and then evenly mixed into the soil.

When plant infected with root-knot nematodes, a series of extensive host plant gene regulation was formed. In the recent studies, several resistance genes (Mi, Ma, rhg1, TIR-NBS-LRR gene) were detected involved in tomato, plum, soybean and peanut, respectively [6, 26-28]. However, what the role of RNAs played in the regulation mechanism of peanut against root-knot nematode was unclear. In this study, a total of 430 differential expressed mRNAs, 80 differential expressed miRNAs, 123 differential expressed cirRNAs and 4453 differential expressed IncRNAs were involved in the process of root-knot nematode regulation mechanism (Fig.S2, Table S1-S5). Meanwhile, the number of differentially expressed genes were 2290 and 555 in resistant genotype and susceptible genotype of Glycine soja under Soybean cyst nematode stress, respectively [29]. A total of 5595 differentially expressed mRNAs responses to nematode infection were detected in peanut. A number of 3178 genes out of 5595 formed three coexpression gene clusters were identified in different genotypes peanut, independent of resistance and susceptibility types [6]. According to the top enrich $\mathrm{GO}$ analysis, the constitutively differentially expressed genes involved in the cell proliferation and stress resistance including cell division, DNA replication initiation, mitosis, stress response, salt stress response and water deprivation, indicating that the genotypes of resistant peanut root had more growth vitality than susceptible peanut under nematode infection [6]. The same results of top enrich GO were analysis in our studies, which were defense response including defense response to bacterium, oomycetes, virus, gram-negative bacterium and fungus and cell proliferation process including DNA binding transcription factor activity (Fig. 6, Fig.S5). In summary, a number of IncRNA, circRNA, miRNA and mRNA showed differentially expressed in response to peanut root-knot nematodes stress, suggesting that RNAs played a crucial role in root morphological changes and regulation mechanism in peanut against root-knot nematodes.

The highlight of miRNA-mediated differentially expressed IncRNA, circRNA and mRNA, the competing endogenous RNA model (Fig. 4) related to root-knot nematode stress response was reconstruction. The target differentially expressed RNAs of miRNA-mediated regulatory network were involved in biological processes of stress resistance including peroxidase activity, lignin biosynthetic process and flavonoid biosynthesis (Fig. 6, Fig.S5, Table S1-S7). The ceRNA network is revealing that when peanut faced to the root-knot nematode stress, the mediator of RNA polymerase II transcription subunit 15-like isoform X1 (NEIN3W) were down-regulated by increased miRNA (gma-MIR482c-p5_2ss12GA19CT). In order to maintain the growth of peanut roots, the one circRNA (circRNA320) and four IncRNAs (MSTRG.2115, MSTRG.30599, MSTRG.30601and MSTRG.31962) formed the competing endogenous RNA model to reduce the inhibition of mRNA by miRNA. Meanwhile, the expression of JF37M9 gene (CLK4-associating serine/arginine rich protein) was increased to keep the peanut development under nematode stress. Anther regulatory network showed that the processes of cold-responsive protein kinase 1-like isoform X4 (DQ3LYR) and G-type lectin S-receptor-like serine/threonine-protein kinase At5g35370 isoform X1 gene 
(42IH8X) were inhibited. With the help of cirRNA (circRNA226) and IncRNA (MSTRG.42738), the synthesis of peroxidase was promoted (S1GD6Q) leading to the free radicals scavenging and damage of peanut roots reducing (Fig. 6, Fig.S3).

Taken together, the integrated analysis of the IncRNA/circRNA-miRNA-mRNA expression profiles could reveal the complex ceRNAs regulatory network and mechanism during peanut root infected by nematodes stress.

\section{Conclusion}

In this study, a total of 430 mRNAs, 111 miRNAs, 4453 IncRNAs and 123 circRNAs were identified differential expression between infected and no-infected peanut through whole transcriptome RNA-seq, respectively. A total of 10 IncRNAs, 4 circRNAs, 5 miRNAs and 13 mRNAs can regularly the expression of mRNA during root-knot nematodes stress by forming competing endogenous RNA and participate in oxidation-reduction process and other various biological metabolism pathways in peanut. The results gained will reveal the role of ceRNAs of peanut in response to root-knot nematodes.

\section{Abbreviations}

ceRNAs: Competing endogenous RNA

P: Phosphorus

\section{Declarations}

\section{Ethics approval and consent to participate}

The author states that the peanuts involved in this study do not involve ethical relations.

\section{Consent for publication}

All authors consent for publication

\section{Availability of data and materials}

Data and materials were provided it in the supplementary Tables 1-7.

\section{Competing interests}

The authors declare that they have no conflict of interest.

\section{Funding}


This research was supported by the National Natural Science Foundation of China (32001575; 32070344; $32001459 ; 31902018$ ) and Natural Science Foundation of Shandong Province (ZR2019PC055, ZR2019PC016, ZR2020QC123).

\section{Authors' contributions}

Conceptual and experiment designs by P. X., Z.L. and X.W, Experiments were conducted by X.W., P.X. G. Z., and S.D; Data analysis performed by X.W., P. X., X. C., and Z. L; Reagents/materials/analysis tools were contributed by P.X., Z. L., and X.C. and the report was written by X.W., P.X., H. L., X. L., and L.Z. All the authors have commented, read and approved the final manuscript.

\section{Acknowledgements}

Not applicable

\section{Authors' information (optional)}

Not applicable

\section{References}

1. Abad P, Gouzy J, Aury J.M, Castagnone-Sereno P, Danchin E.G.J, Deleury E, Perfus-Barbeoch L, Anthouard V, Artiguenave F, Blok V.C, et al. Genome sequence of the metazoan plant-parasitic nematode Meloidogyne incognita. Nat. Biotechnol. 2008; 26(8): 909.

2. Timper P. Utilization of biological control for managing plant-parasitic nematodes. Bioligical Cotrol of Plant-Parasitic Nematades: Progress in Biological Control. 2011; 11: 259-289.

3. Wang H, Shi Y.M, Ren Y, Li S.L, Jiao K, Yuan M, Li H.J, Development of SSR markers for root-knot nematode resistance in peanut. Journal of Peanut Science. 2008; 37(2): 14-17.

4. Dong W, Holbrook C.C, Timper P, Brenneman T.B, Mullinix B.G, Comparison of methods for assessing resistance to Meloidogyne arenaria in peanut. J Nematol. 2007; 39(2): 169-175.

5. Zhuang W, Chen H, Yang M, Wang J, Pandey M.K, Zhang C, Chang W.C, Zhang L, Zhang X, Tang R, et al. The genome of cultivated peanut provides insight into legume karyotypes. polyploid evolution and crop domestication. Nat. Genet. 2019; 51 865-876.

6. Josh C, Ye C, Larissa A. G, Thiago M, David B, Soraya L. B, Patricia T. C, Corley H, Peggy O. A. Gene expression profiling describes the genetic regulation of meloidogyne arenaria resistance in Arachis hypogaea and reveals a candidate gene for resistance. Sci. Rep. 2017; 7(1); 1317.

7. Wang J, Yu W, Yang Y, Li X, Chen T, Liu T, Ma N, Yang X, Liu R, Zhang B. Genome-wide analysis of tomato long non-coding RNAs and identification as endogenous target mimic for microRNA in response to TYLCV infection. Sci. Rep. 2015; 5:16946.

8. Schneider T; Bindereif A. Circular rnas coding or noncoding? Cell Res. 2017; 27(6): 724-725. 
9. Wang Y, GaoL, Zhu B, Zhu H, Luo Y, Wang Q, Zuo J. Integrative analysis of long non-coding RNA acting as ceRNAs involved in chilling injury in tomato fruit. Gene. 2018; 667: 25-33.

10. Fabian M.R, Sundermeier T.R, Sonenberg N. Understanding how miRNAs post-transcriptionally regulate gene expression. Prog. Mol. Subcell Biol. 2010; 50: 1-20.

11. Lee S, Lee W, Ren S, Han K, A Method for Constructing an Integrative Network of Competing Endogenous RNAs. Intelligent Computing Theories and Application. 2021; 407-420.

12. Khraiwesh B, Zhu J, Zhu J. Role of miRNAs and siRNAs in biotic and abiotic stress responses of plants. Biochim. Biophys. Acta-biomembranes. 2012; 1819: 137-148.

13. Boccara M, Sarazin A, Thiébeauld O, Jay F, Voinnet O, Navarro L, Colot V. The Arabidopsis miR472RDR6 silencing pathway modulates PAMP-and effector-triggered immunity through the posttranscriptional control of disease resistance genes. PLoS Pathog. 2014; 10e1003883.

14. Li Y, Lu Y.G, Shi Y, Wu L, Xu Y.J, Huang F, Guo X.Y, Zhang Y, Fan J, et al. Multiple rice microRNAs are involved in immunity against the blast fungus Magnaporthe oryzae. Plant Physiol. 2014; 164: 10771092.

15. Zhao C, Li T, Zhao Y, Zhang B, Wang X. Integrated small RNA and mRNA expression profiles reveal miRNAs and their target genes in response to Aspergillus flavus growth in peanut seeds. BMC Plant Biology. 2020; 20(1): 215.

16. Zhang X, Ma X, Ning L, Li Z, Yin D. Genome-wide identification of circular RNAs in peanut (Arachis hypogaea L.). BMC Genomics. 2019; 20(1): 653.

17. Pan T, Sun X, Liu Y, Li H, Deng G, Lin H, Wang S. Correction to heat stress alters genome-wide profiles of circular RNAs in Arabidopsis. Plant Mol Biol. 2018; 96(3): 231-231.

18. Sun Z, Huang K, Han Z, Wang P, Fang Y. Genome-wide identification of Arabidopsis long noncoding RNAs in response to the blue light. Sci. Rep. 2020; 10(1): 6229.

19. Song X, Hu J, Wu T, Yang Q, Feng X, Li H, Feng S, Cui C, Yu Y, Zhou R, et al.. Comparative analysis of long noncoding RNAs in angiosperms and characterization of long noncoding RNAs in response to heat stress in Chinese cabbage. Hortic. Res. 2021; 8(1): 48.

20. Ma P, Zhang X, Luo B, Chen Z, He X, Zhang H, Li B, Liu D, Wu L, Gao S, et al. Transcriptomic and genome-wide association study reveal long noncoding RNAs responding to nitrogen deficiency in maize. BMC Plant Biol. 2021; 21(1): 93.

21. Fan C, Hao Z, Yan J, Li G. Genome-wide identification and functional analysis of lincRNAs acting as miRNA targets or decoys in maize. BMC Genomics. 2015; 16(1): 793.

22. Lu Q, Guo F, Xu Q, Jing C. LncRNA improves cold resistance of winter wheat by interacting with miR398. Funct Plant Biol. 2020; 47(6): 544-557.

23. Zhai R, Ye S, Zhu G, Lu Y, Ye J, Yu F, Chu Q, Zhang X. Identification and integrated analysis of glyphosate stress-responsive microRNAs IncRNAs and mRNAs in rice using genome-wide highthroughput sequencing. BMC Genomics. 2020; 21(1): 238. 
24. Pandey R.K. Physiological and biochemical changes in susceptible and resistant rice cultivars induced by root-knot nematode. Meloidogyne graminicola. Indian Phytopathology. 2020; 73(2): 321328.

25. Elhady A, Hallmann J, Heuer H. Symbiosis of soybean with nitrogen fixing bacteria affected by root lesion nematodes in a density-dependent manner. Sci. Rep. 2020; 10(1): 1619.

26. Milligan S.B, Bodeau J, Yaghoobi J, Kaloshian I, Zabel P, Williamson V.M. The root knot nematode resistance gene $\mathrm{Mi}$ from tomato is a member of the leucine zipper nucleotide binding leucine-rich repeat family of plant genes. Plant Cell. 1998; 10: 1307-1319.

27. Claverie M, Dirlewanger E, Bosselut N; Ghelder C.V, Voisin R, Kleinhentz M, Lafargue B, Abad P, Rosso M.N, Chalhoub B, Esmenjaud D. The Ma gene for complete-spectrum resistance to Meloidogyne species in Prunus is a TNL with a huge repeated C-terminal post-LRR region. Plant Physiol. 2011; 156: $779-792$.

28. CookD.E, Lee T.G, Guo X, Melito S, Wang K, Bayless A.M, Wang J, Hughes T.J, Willis D.K, Clemente T.E, et al. Copy number variation of multiple genes at $R h g 1$ mediates nematode resistance in soybean. Science. 2012; 338 (6111): 1206-1209.

29. Zhang H, Kjemtrup-Lovelace S, Li C, Luo Y, Chen L.P, Song B.H. Comparative RNA-Seq Analysis Uncovers a Complex Regulatory Network for Soybean Cyst Nematode Resistance in Wild Soybean (Glycine soja).Sci. Rep. 2017; 7(1):9699.

30. Xu P, Lv Z, Zhang X, Wang X, Pu Y, Wang H, Yi B, Wen J, Ma C, Tu J, et al. Identification of molecular markers linked to trilocular gene (mc1) in Brassica juncea L. Mol. Breeding. 2014; 3: 425-434.

31. Wang X, Chen Y, Thomas C.L, Ding G, Xu P, Shi D, Grandke F, Jin K, Cai H, Xu F, et al. Genetic variants associated with root system architecture of oilseed rape under contrasting phosphate availabilities through genome -wide analyses. DNA Res. 2017; 24(4): 407-417.

32. Chen H, Yang Q, Chen K, Zhao S,. Zhang C, Pan R, Cai T, Deng Y, Wang X, Chen Y, et al. Integrated microRNA and transcriptome profiling reveals a miRNA-mediated regulatory network of embryo abortion under calcium deficiency in peanut (Arachis hypogaea L.).BMC Genomics. 2019; 20(1): 392.

33. Li D, Chen W, Luo L, Wang Y, Shang J, Zhang Y, Chen G, Li S. Prospective IncRNA-miRNA-mRNA regulatory network of long non-coding RNA LINC00968 in non-small cell lung cancer A549 cells: A miRNA microarray and bioinformatics investigation. Int. J. Mol. Med. 2017; 40(6):1895-1906.

\section{Tables}

Table 1. The difference root morphology types between no-infected and infected peanut caused by rootknot nematodes. 


\begin{tabular}{lll} 
Traits & No-infected peanut & Infected peanut \\
\hline Root-knot nematode number $(\mathrm{n})$ & 0 & $21.33 \pm 4.16^{\star \star}$ \\
\hline Primary root length $(\mathrm{cm})$ & $28.26 \pm 1.38^{\star \star}$ & $25.06 \pm 1.79$ \\
\hline Lateral root number $(\mathrm{n})$ & $41 \pm 3$ & $52.33 \pm 5.13^{\star}$ \\
\hline Total root surface area $\left(\mathrm{cm}^{2}\right)$ & $644.94 \pm 21.53^{\star \star}$ & $478.36 \pm 42.96$ \\
\hline Root angle $\left(^{\circ}\right)$ & $106.58 \pm 8.94^{\star}$ & $79.26 \pm 7.47$ \\
\hline Lateral root density $(\mathrm{cm} / \mathrm{n})$ & $0.6902 \pm 0.0208^{\star \star}$ & $0.4801 \pm 0.0263$
\end{tabular}

Note: $\mathrm{P}<0.01$ is considered as highly significant and labeled as ${ }^{* *}$. $\mathrm{P}<0.05$ is considered as significant and labeled as *.

\section{Figures}




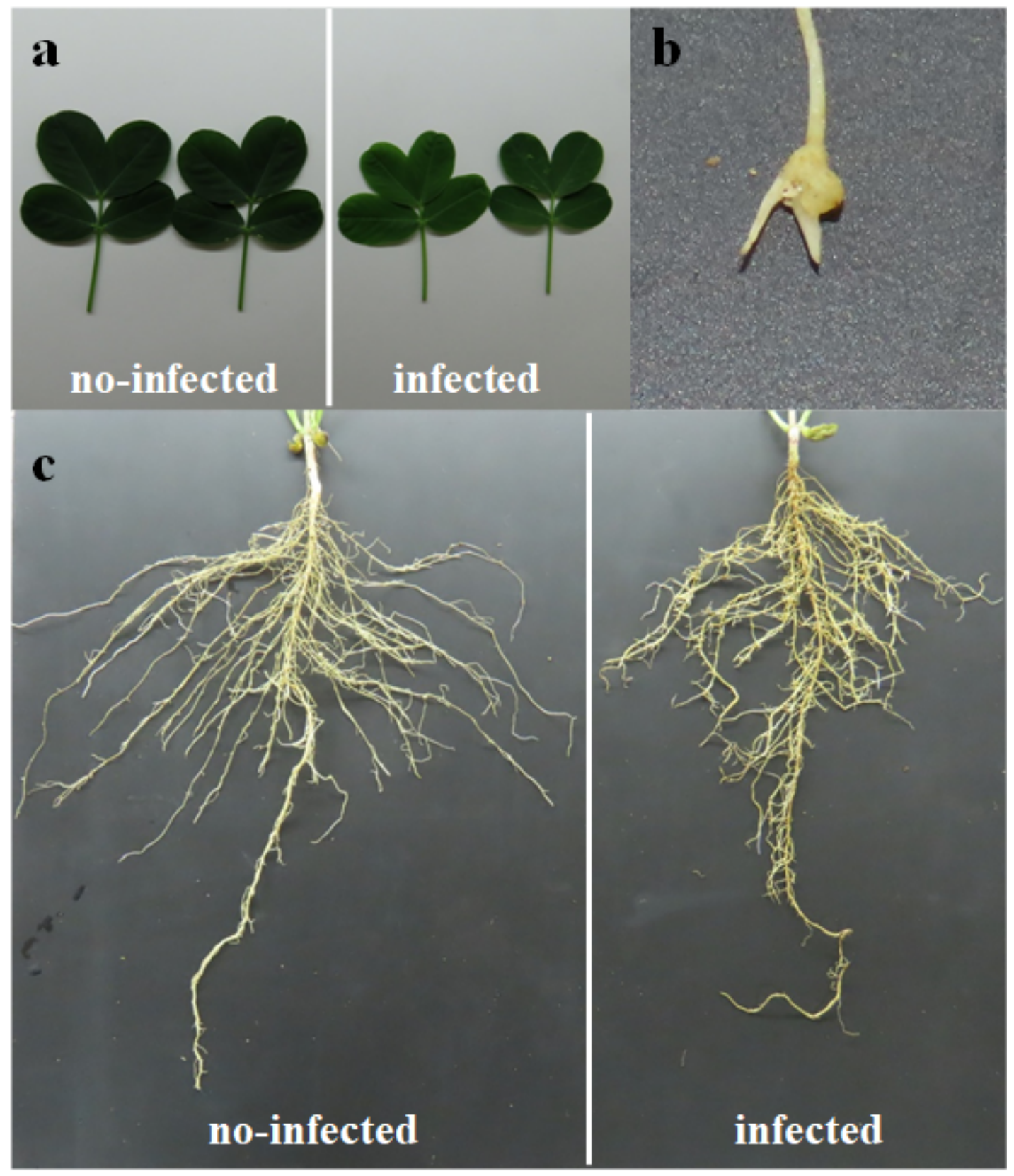

\section{Figure 1}

The infected and no-infected peanut (Huayu22) phenotype. a, The different colors and size leaf between no-infected and infected peanut. b, The peanut root-knot. c, The root morphology types caused by rootknot nematodes. 

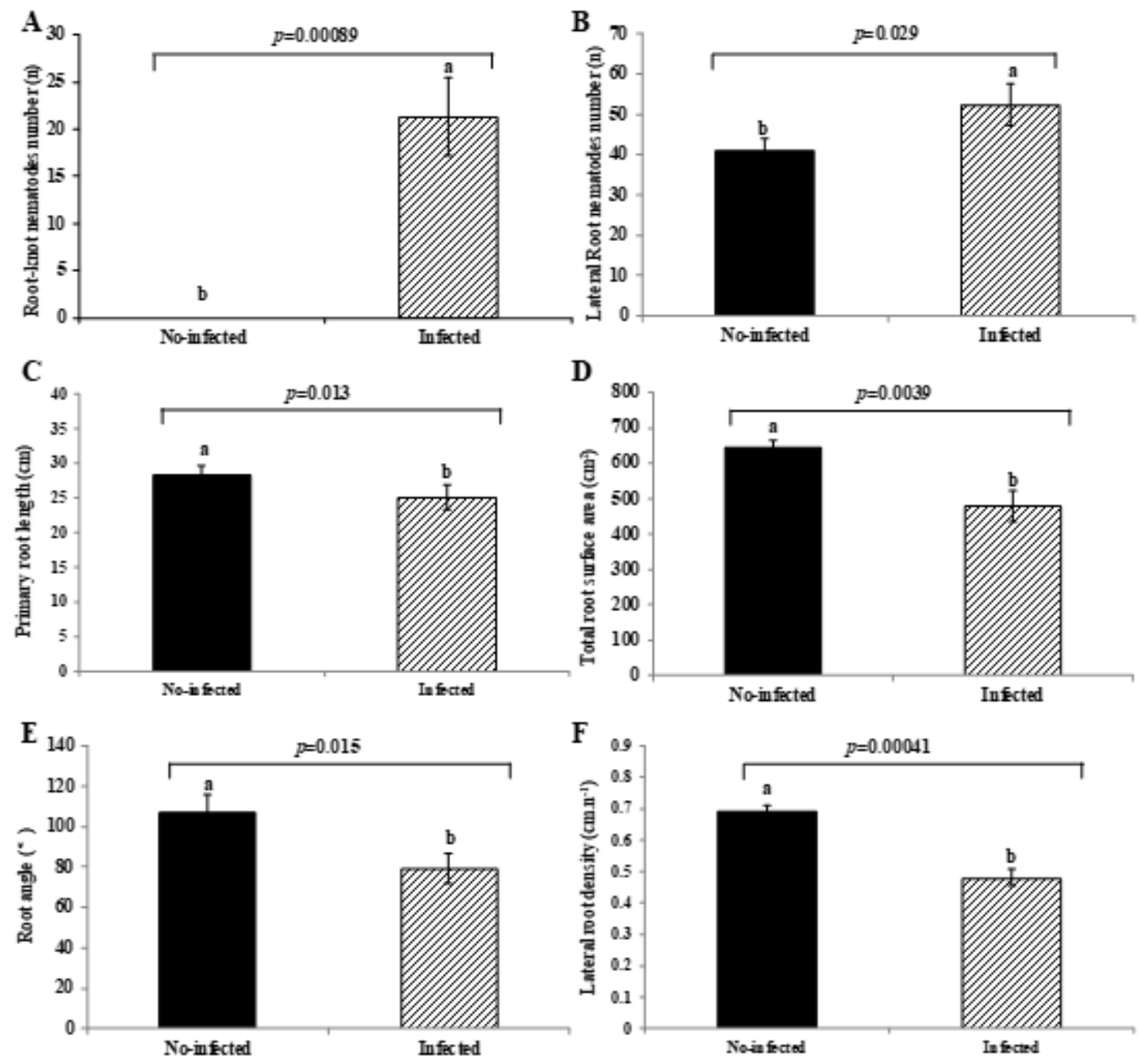

Figure 2

The no-infected and infected peanut root morphology traits caused by root-knot nematodes.
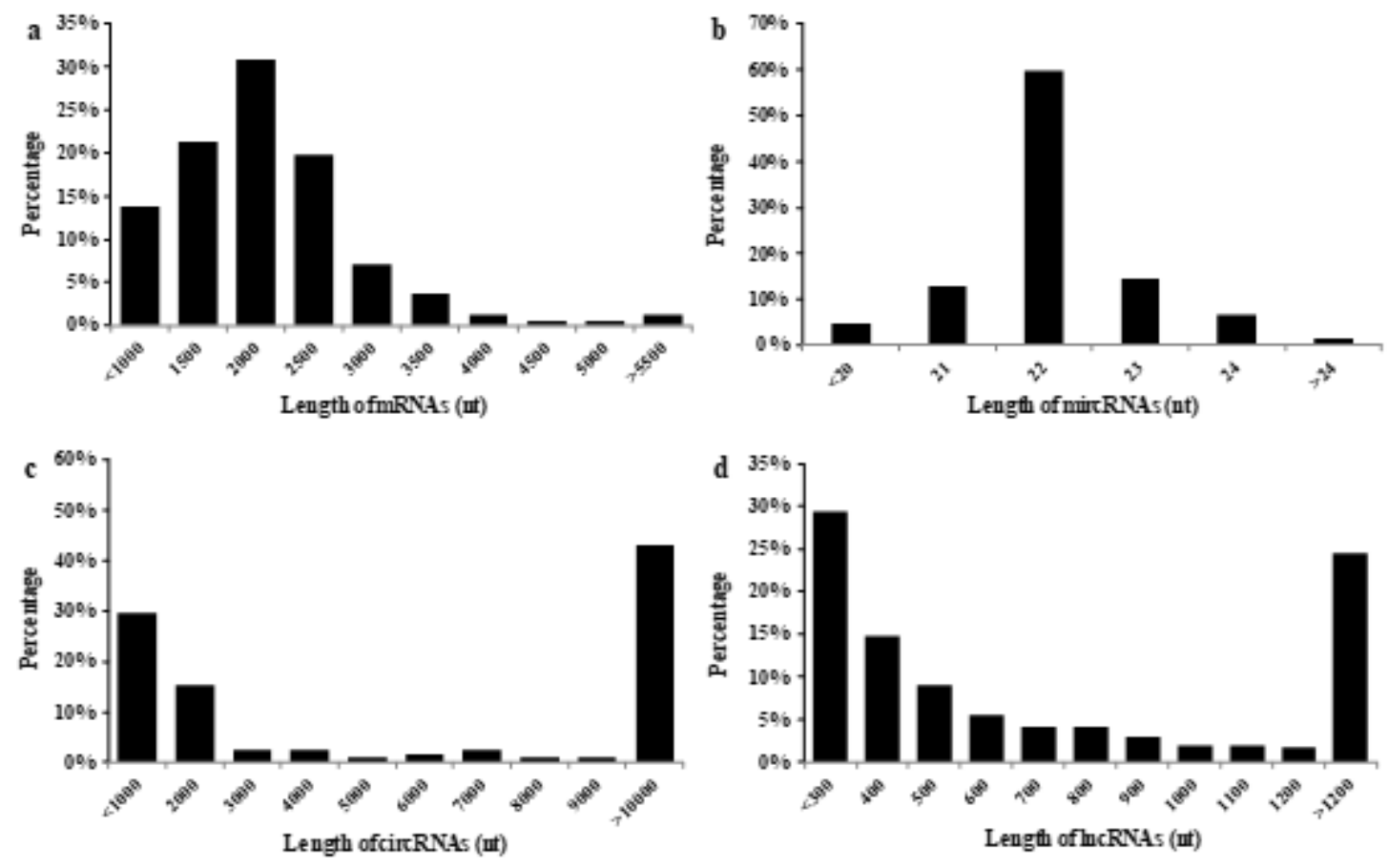


\section{Figure 3}

Sequence length distribution of differential expressed mRNAs, miRNAs, circRNAs and IncRNAs. a, The length of differential expressed mRNAs. $b$, The length of differential expressed miRNAs. $c$, The length of differential expressed circRNAs. $d$, The length of differential expressed IncRNAs.

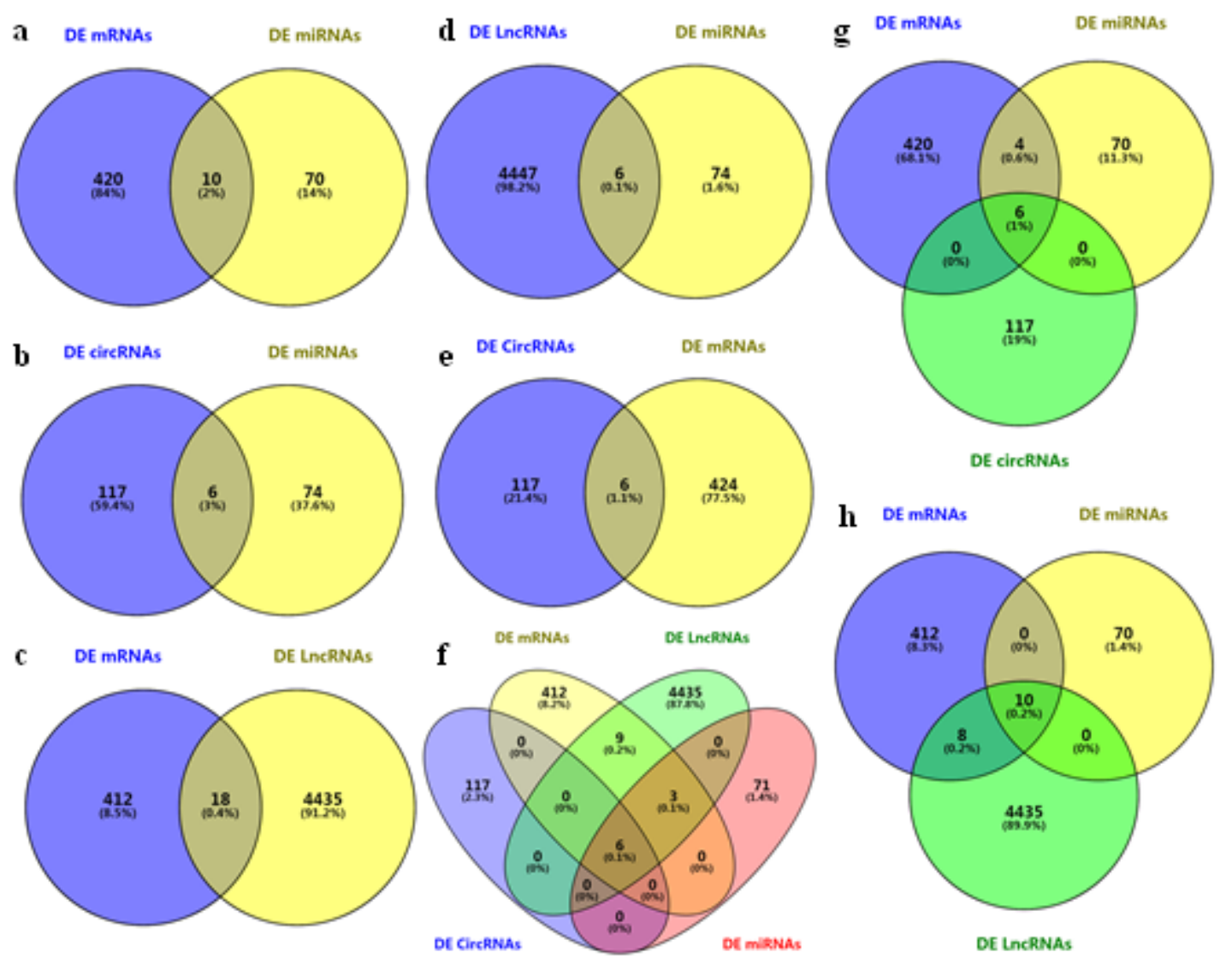

Figure 4

The number of differential expressed mRNAs, miRNAs, circRNAs, IncRNAs and the construction of regulatory networks. a, The differential expressed miRNAs and the target mRNAs. $b$, The differential expressed miRNAs and the target circRNAs. c, The co-expressed differential expressed mRNAs and the IncRNAs. $d$, The differential expressed miRNAs and the target IncRNAs. e, The co-expressed differential expressed mRNAs and the circRNAs. $f$, The ceRNAs regulatory network contains IncRNA/circRNA-miRNAmRNA. $g$, The mRNA-miRNAs-circRNAs regulator network. $h$, The mRNA-miRNAs-IncRNAs regulator network. 


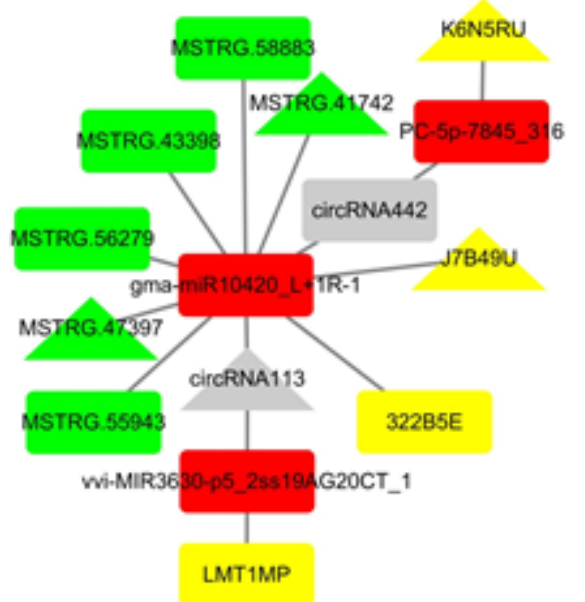

mRNA

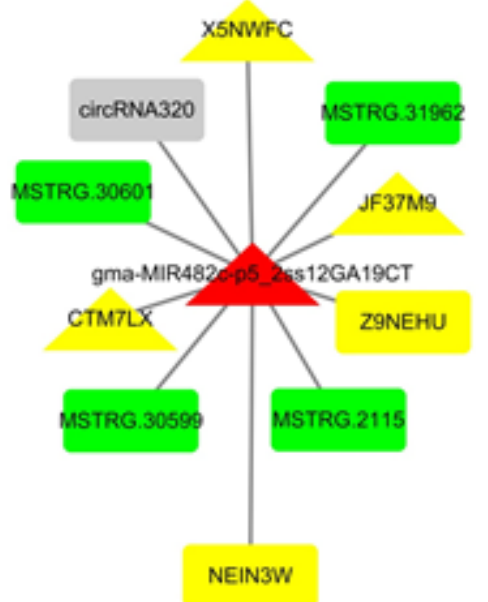

circRNA

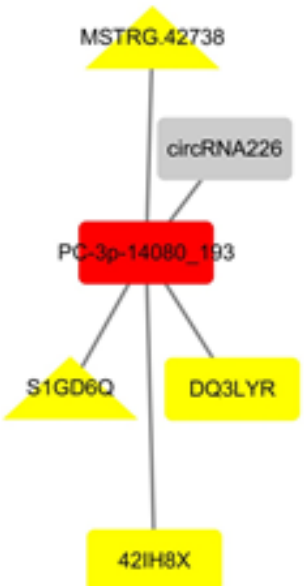

up-reguhr

down-regular

\section{Figure 5}

The regulatory ceRNA network of IncRNA/circRNA-miRNA-mRNA in response to root-knot nematode stress.

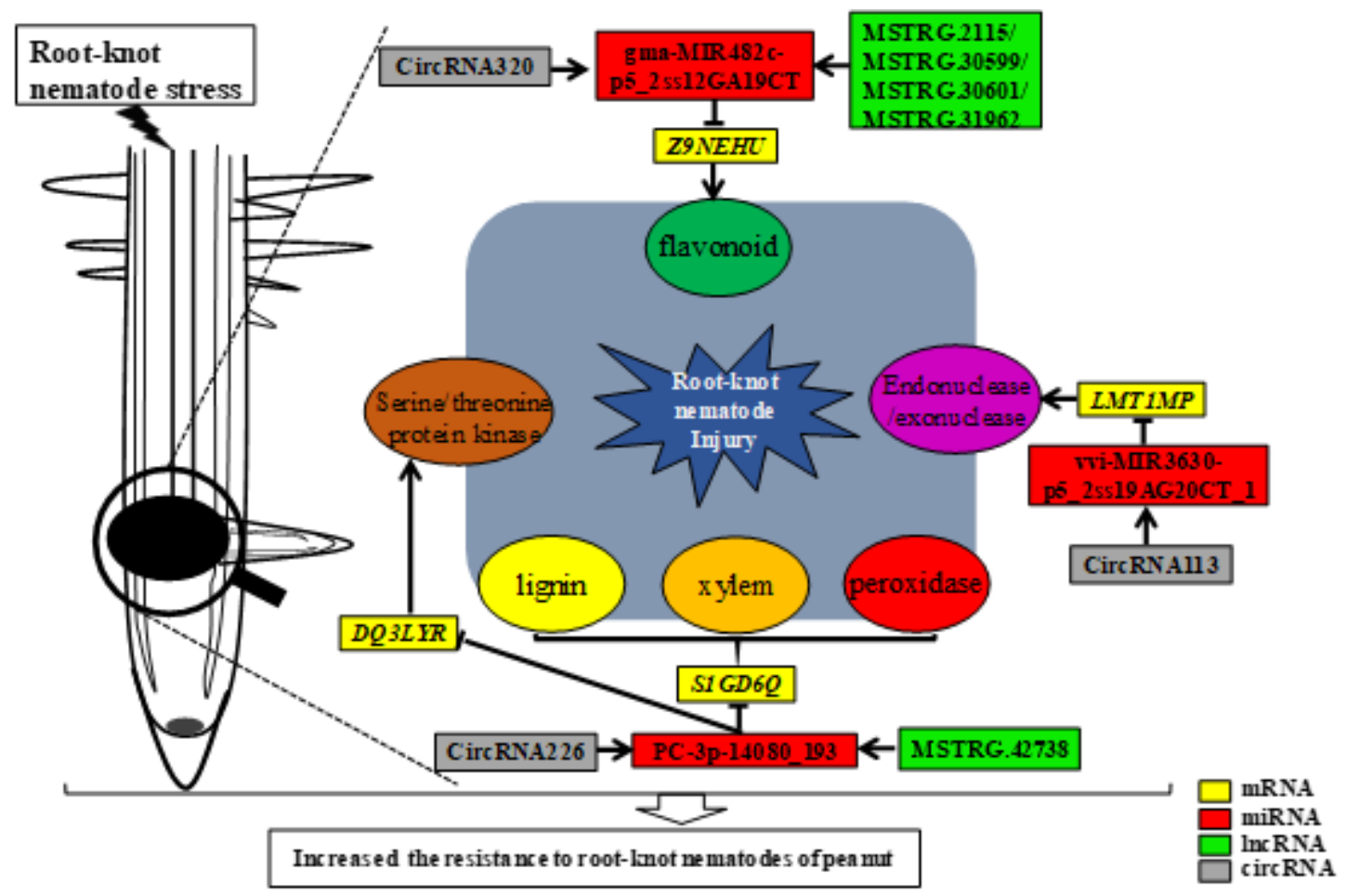

\section{Figure 6}

Regulator network model of the miRNAs-mediated IncRNA, circRNA and mRNA involved in root-knot nematode stress.

\section{Supplementary Files}


This is a list of supplementary files associated with this preprint. Click to download.

- SupplementaryFigure1.xls

- SupplementaryFigure2.docx

- SupplementaryFigure3.pptx

- SupplementaryFigure4.xls

- SupplementaryFigure5.docx

- SupplementaryTable1.xlsx

- SupplementaryTable2.xlsx

- SupplementaryTable3.xlsx

- SupplementaryTable4.xIsx

- SupplementaryTable5.xlsx

- SupplementaryTable6.xlsx

- SupplementaryTable7.xlsx 